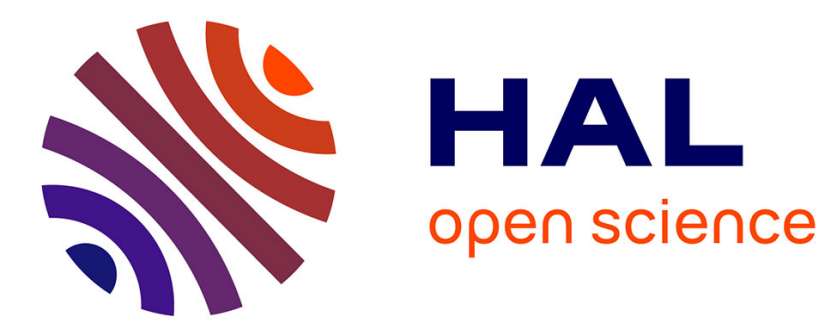

\title{
On-line MR imaging for dose validation of abdominal radiotherapy
}

\author{
Markus Glitzner, Sjoerd Crijns, Baudouin Denis de Senneville, Charis \\ Kontaxis, Fieke Prins, Jan Lagendijk, Bas Raaymakers
}

\section{- To cite this version:}

Markus Glitzner, Sjoerd Crijns, Baudouin Denis de Senneville, Charis Kontaxis, Fieke Prins, et al.. On-line MR imaging for dose validation of abdominal radiotherapy. Physics in Medicine and Biology, 2015. hal-01211405

\author{
HAL Id: hal-01211405 \\ https://hal.science/hal-01211405
}

Submitted on 5 Oct 2015

HAL is a multi-disciplinary open access archive for the deposit and dissemination of scientific research documents, whether they are published or not. The documents may come from teaching and research institutions in France or abroad, or from public or private research centers.
L'archive ouverte pluridisciplinaire HAL, est destinée au dépôt et à la diffusion de documents scientifiques de niveau recherche, publiés ou non, émanant des établissements d'enseignement et de recherche français ou étrangers, des laboratoires publics ou privés. 


\title{
On-line MR Imaging for Dose Validation of Abdominal Radiotherapy
}

\author{
M Glitzner ${ }^{1}$, S P M Crijns ${ }^{1}$, B Denis de Senneville ${ }^{1,2}$, \\ C Kontaxis ${ }^{1}$, F M Prins ${ }^{1}$, J J W Lagendijk ${ }^{1}$, \\ B W Raaymakers ${ }^{1}$ \\ ${ }^{1}$ Department of Radiotherapy, University Medical Center Utrecht, \\ Heidelberglaan 100, 3584 CX Utrecht, the Netherlands \\ 2 Mathematical Institute of Bordeaux, UMR 5251 CNRS/University of \\ Bordeaux, 33405 Talence Cedex, France \\ E-mail: m.glitzner@umcutrecht.nl
}

\begin{abstract}
.
For quality assurance and adaptive radiotherapy, validation of the actual delivered dose is crucial.

Intrafractional anatomy changes cannot be captured satisfactorily during treatment with hitherto available imaging modalitites. Consequently, dose calculations are based on the assumption of static anatomy throughout the treatment. However, intra- and interfraction anatomy is dynamic and changes can be significant. In particular, hypofractionated and escalated radiotherapy thus demand for reliable dose reconstruction based on periodic imaging.

In this paper, we investigate the use of an MR-linac as a dose tracking modality for the validation of treatments in abdominal targets where both respiratory and long-term peristaltic and drift motion occurs.

The on-line MR imaging capabilities of the modality provides the means to perform respiratory gating of both delivery and acquisition yielding a model-free respiratory motion management under free breathing conditions.

In parallel to the treatment, the volumetric patient anatomy was captured and used to calculate the applied dose. Subsequently, the individual doses were warped back to the planing grid to obtain the actual dose accumulated over the entire treatment duration. Eventually, the planned dose was validated by comparison with the accumulated dose.

Representatively for a site subject to breathing modulation, two kidney cases (25Gy target dose) demonstrated the working principle on volunteer data and simulated delivery. The proposed workflow successfully showed its ability to track local dosimetric changes. Integration of the on-line anatomy information could reveal local dose variations -2.3 to $1.5 G y$ in the target volume of a volunteer dataset. In the adjacent organs at risk, high local dose errors ranging from -2.5 to $1.9 G y$ could be traced back.
\end{abstract}

Keywords: respiratory motion compensation, dose reconstruction, MR guidance, adaptive radiotherapy, hypofractionated treatment, local deformations

Submitted to: Phys. Med. Biol. 


\section{Introduction}

In an attempt for higher tumor control and better organ at risk (OAR) sparing, new motion management strategies are gaining interest.

Traditionally, geometric margin concepts such as planning target volume (PTV), internal target volume (ITV) (ICRU 2010) or mid-ventilation (Wolthaus et al. 2006) approaches for uncertainty compensation are used. Additionally, various active motion compensation methods have been recently investigated. Amongst them are guided patient positioning (Borst et al. 2007) as well as real-time beam gating (Keall, Vedam, George, Bartee, Siebers, Lerma, Weiss \& Chung 2006, Shirato et al. 2000) and tracking techniques (Keall et al. 2001, Keall, Colvill, O'Brien, Ng, Poulsen, Eade, Kneebone \& Booth 2014, Crijns et al. 2012). While the former are mostly X-ray based imaging techniques, the latter are controlled by feedback variables extracted from external or internal surrogates, which ideally move in phase with the target volume (Ruan et al. 2011). Obviously, this in-phase condition is non-trivial to maintain generally, as system lag and imaging uncertainties introduce position errors (Keall, Cattell, Pokhrel, Dieterich, Wong, Murphy, Vedam, Wijesooriya \& Mohan 2006, Glitzner et al. 2015). Furthermore, using target surrogates for target tracking, motion of surrounding tissue in the beam's path is entirely neglected. Additionally, although several authors have previously investigated dose tracking using surrogate signals (e.g. (Poulsen et al. 2012, Ravkilde et al. 2014)), the assumed (rigid) translations of the entire patient volume in phase with the surrogate signal are not physiologically sound.

The fusion of magnetic resonance imaging (MRI) diagnostics and radiotherapy treatment (Lagendijk et al. 2014, Fallone 2014, Mutic \& Dempsey 2014, Keall, Barton \& Crozier 2014) enables new options for motion management. With these combined modalitites, volumetric information is available on-line, i.e. during irradiation.

This $3 D$ information can serve for both target tracking (plan adaptation) and dose tracking (plan validation) (Kupelian \& Sonke 2014). However, although MRI

offers unprecedented soft-tissue contrast, volumetric imaging times are in the order of seconds. In the abdomen, real-time MRI in $3 D$ is thus technically challenging, due to the high mobility of the abdominal organs (Langen \& Jones 2001, Bussels et al. 2003) causing motion induced artifacts.

The aspiration of this work is to develop a technique to enable joint respiratory motion compensation and detailed $3 D$ plan validation for the treatment of highly mobile abdominal organs using an magnetic resonance (MR)-linac modality. Therefore, the presented method covers two aspects, i.e.

- apply respiratory motion compensation using responsive, real-time radiation gating on an internal surrogate (diaphragm) and

- recover the actual dose deposition by using $3 D$ imaging over the active treatment time.

The unique combination of these fundamentals enables respiratory motion compensated, quality-assessed radiotherapy of abdominal sites. As a proof of concept, we evaluated two (virtual) kidney cases. This site was chosen because of its high mobility (Schwartz et al. 1994, Stam, van Vulpen, Barendrecht, Zonnenberg, Intven, Crijns, Lagendijk \& Raaymakers 2013) during respiration and its expected benefit from hypofractionated treatments (De Meerleer et al. 2014). Planned and true dose depositions were calculated and compared offline, integrating recorded treatment machine parameters, MR-acquired images and deformable image registration. 


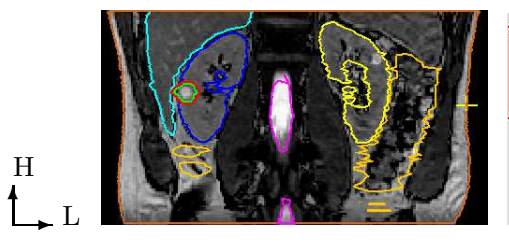

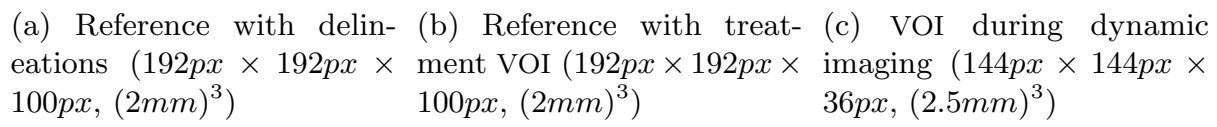

Figure 1: Planning images ( $\mathrm{a}$ and $\mathrm{b}$ ) and intrafraction images $(\mathrm{c})$. Kidneys, liver, myelum, pancreas and bowels were delineated in addition to the GTV (green) and PTV (red). In the depicted anatomy, a benign cyst was delineated as target volume in the kidney.

Anatomic directions: head $(\mathrm{H})$ and left $(\mathrm{L})$.

\section{Methods}

In this paper, rapidly sampled MR-navigator echoes were used to sample the respiration and restrict both the treatment beam and the image acquisition to the quasi-static expiration phase of the breathing cycle (Section 2.1). During the gating interval, delivery took place and a fast $3 D$ images were taken from the target region and its entire surrounding, i.e. all OAR in the beam's path. Due to the respiratory motion compensation, the images contained solely cycle-to-cycle and long-term variation, such as spontaneous and drift motion. Motion estimations (sections 2.2 and 2.3) extracted from the $3 D$ imaging volumina are then used to recover anatomy variations in the course of treatment.

By continuous logging during delivery of the generated plan (sections 2.4 and 2.5) the individual anatomies could be correlated with the machine state at every instance. Subsequently, applying Monte-Carlo (MC) dose calculations (section 2.7) on the pseudo-computed tomographys (CTs) of all individual anatomies (section 2.6), the actual delivered dose was reconstructed. In this way, an iterative dose accumulation was performed over the entire treatment time.

\subsection{MRI acquisition}

All imaging experiments were performed on a 1.5T MRI machine (Ingenia, Philips Medical Systems, Best, The Netherlands) using a 28-channel torso array.

Two different MR pulse sequences were developed. Both of them employed navigator echoes (Wang et al. 1996) for respiratory gating in the exhale phase of the respiratory cycle. The navigator beam was placed on the right liver dome in order to maximize sensitivity to breathing excursions in caudo-cranial (CC) direction.

The images from the first pulse sequence were used for generating the delineation reference (figure $1 \mathrm{a}$ and $1 \mathrm{~b}$ ). The sequence consisted of navigator-gated, balanced turbo field echo (TFE) shots which were triggered in a narrow gating window of $2 \mathrm{~mm}$ (exhalation) in order to maximally suppress breathing artifacts. The shot length was approximately $250 \mathrm{~ms}$ including an $\alpha / 2$-instance and 10 additional startup cycles to equilibrate the MR signal.

For fast $3 D$ imaging during treatment a second sequence was developed. It 
consisted of a navigator echo ( $5 \mathrm{~mm}$ gating window at exhalation) followed by a binomial, water-selective pulse. Subsequently, a multi-shot echo planar imaging (EPI) readout was driven to increase sampling efficiency due to the long navigator and water-selective preparation. The long water-selective pulse was chosen to enhance the performance of the image registration algorithm used (Section 2.2) which greatly benefited from suppressed fat signals.

The EPI readout is prone to geometric distortions due to susceptibility variations (Haacke et al. 1999). Thus, the EPI train length was limited to $21 k$-lines per shot to lower image acquisition times to around $3 s$. Additionally, moderate sensitivity encoding (SENSE) was employed with undersampling factors of $P=1.5$ and $S=1.5$ in the anterior-posterior (AP) and CC phase encoding direction, respectively.

The sequence parameters were $T_{R} / T_{E} / \alpha=47 \mathrm{~ms} / 9.8 \mathrm{~ms} / 25^{\circ}$. Typical gradient characteristics reported by the system were $192 \mathrm{~T} / \mathrm{m} / \mathrm{s}$ for the slew rates, $12 \mathrm{mT} / \mathrm{m}$ EPI-blip strength and $22 m T / m$ readout strength.

As volume of interest (VOI), a $10 \mathrm{~cm}$ thick transverse slab was selected (figure 1c) to cover the lower liver/upper right kidney and thus the treatment volume and its surroundings with $(2.5 \mathrm{~mm})^{3}$ isotropic resolution.

\subsection{Motion estimation}

The isotropic $3 D$ data MRI acquired while treatment is inherently suited for non-rigid image registration. In this work an implementation of optical flow-based registration (Roujol et al. 2010) was employed. The optical flow's objective function by Horn \& Schunck (1981) reads,

$$
\iiint_{\Omega}\left(I_{x} v_{x}+I_{y} v_{y}+I_{z} v_{z}+I_{t}\right)^{2}+\alpha^{2}\left(\left\|\nabla v_{x}\right\|_{2}^{2}+\left\|\nabla v_{y}\right\|_{2}^{2}+\left\|\nabla v_{z}\right\|_{2}^{2}\right) d x d y d z
$$

with $I_{t}, I_{x}, I_{y}$ and $I_{z}$ being the temporal and spatial derivatives. Next to the motion estimation using image gradients (left side of (1)) a data regularization term with a single parameter $\alpha$ penalizes sudden spatial changes of the estimated motion $\left(\nabla v_{x}, \nabla v_{y}, \nabla v_{z}\right)$. This regularization has been introduced for therapy guidance on mobile organs (Denis De Senneville et al. 2007) and validated for organ deformations (Østergaard Noe et al. 2008). Next to the single parameter, a further advantage is the performance of the optical flow algorithm when implemented on graphics processing units (GPUs)(Roujol et al. 2010). In this work the regularization parameter $\alpha$, was set to a conservative $\alpha=0.3$, in order to avoid non-continuous motion estimates (Roujol et al. 2011). Motion estimation of the continuously acquired volumes $(144 p x \times 144 p x \times 36 p x)$ took approximately $230 m s$ using a compute unified device architecture (CUDA) implementation on an Geforce GTX280 (NVIDIA, Santa Clara, CA, USA). The host computer was equipped with 2 central processing units (CPUs) (Penryn quad-core, Intel, Santa Clara, CA, USA) with 8GB DDR3 SDRAM memory.

\subsection{Gridding strategies}

Generally, motion is estimated between two dynamics: One is the reference $\left(I_{\text {ref }}\right)$ holding the initial anatomy. The second $\left(I_{n}\right)$ is a version of the reference, which underwent physiologic deformation.

When estimating motion, generally, a pull-back deformation vector field (DVF) $V_{i}(\mathbf{x})$ is calculated for each dynamic image $I_{i}(\mathbf{x})$, so that 


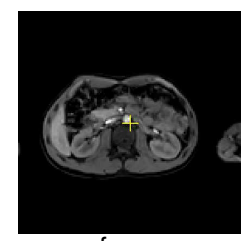

reference

$$
\left(V_{n}\right)^{-1} \downarrow \uparrow V_{n}
$$

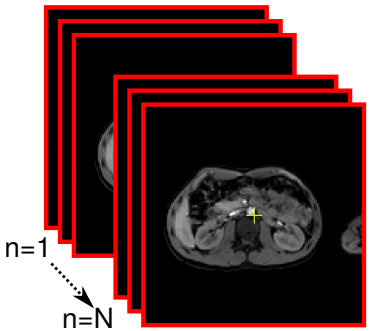

(a) DVF estimation

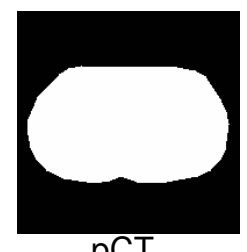

pCT $\downarrow\left(V_{n}\right)^{-1}$

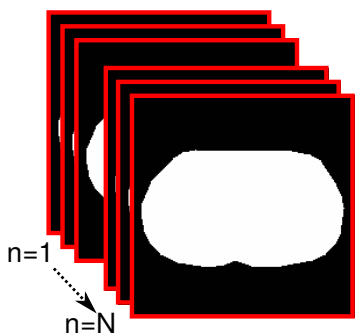

(b) pCT generation

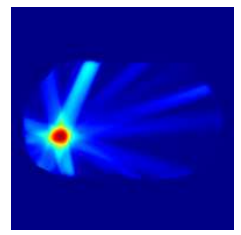

$\Sigma \uparrow \vee_{n}$

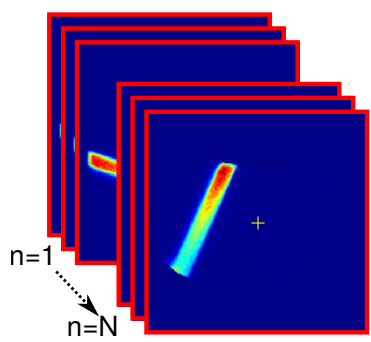

(c) accumulation

Figure 2: DVF estimations (a) and regridding for pCT-generation (b) and dose accumulation (c).

$$
\mathbf{I}_{r e f}(\mathbf{x}) \simeq \mathbf{I}_{n}\left(\mathbf{x}+V_{n}(\mathbf{x})\right) .
$$

This projects the content of the $n^{\text {th }}$ dynamic's grid to the reference grid. In this work, this transformation is consequently used to warp dose deposited on an intermediate grid back onto the reference. Is it accumulated on the reference grid (figure 2c).

Contrary, the push-forward DVF is transforming content from the reference grid onto the dynamic grid, i.e.

$$
\mathbf{I}_{n}(\mathbf{x}) \simeq \mathbf{I}_{\text {ref }}\left(\mathbf{x}+V_{n}^{-1}(\mathbf{x})\right) .
$$

Herein, as illustrated in figure 2b, this transformation is employed to construct dynamic CT data corresponding to the anatomical changes measured by the continuous $3 D$ MRI as proposed by Boye et al. (2013).

Figure 3 depicts the difference between push-forward and pull-back DVF. The latter can be estimated by interchanging reference and dynamic image in the motion estimation algorithm. Ideally, $V_{n}$ and $V_{n}^{-1}$ are inversely consistent (Yang et al. 2008) such that

$$
V_{n}\left(\mathbf{x}+V_{n}^{-1}(\mathbf{x})\right)+V_{n}^{-1}(\mathbf{x})=0 .
$$

In practice however, inverse consistency is generally not achieved by motion estimation algorithms. Thus, in this paper, inverse consistency was approximated by an iterative solution previously described in Heinrich et al. (2012) after calculating both push and pull DVF. 

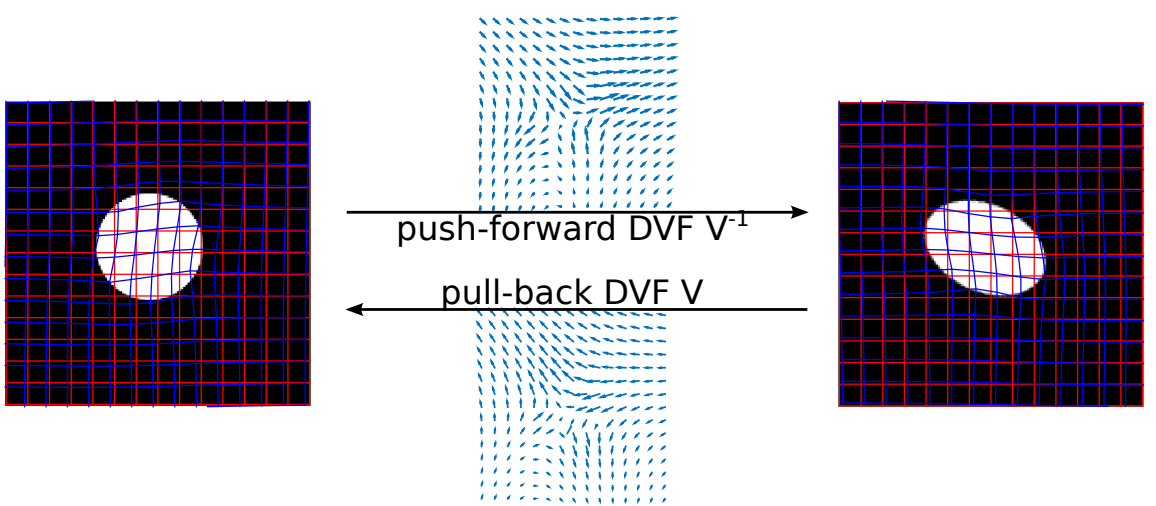

Figure 3: warping between grids; The pull-back DVF provides the mapping back to initial grid of the circle. The inverse, $V^{-1}$, serves to reconstruct values from the circle's grid onto the ellipse's grid. Deformed indexing grids are shown in blue. Arrows indicate the direction of registration.

\begin{tabular}{lll} 
Site & Parameter & Value \\
\hline GTV & $D_{99}$ & $20 G y$ \\
\hline Kidney & $D_{25}$ & $\leq 5 G y$ \\
& $D_{\text {mean }}$ & $\leq 8 G y$ \\
\hline Liver & $D_{30}$ & $\leq 2.5 G y$ \\
\hline Bowels & $D_{5}$ & $\leq 20 G y$ \\
& $D_{\max }$ & $\leq 21 G y$ \\
\hline Stomach & $D_{4}$ & $\leq 22.5 G y$ \\
\hline Myelum & $D_{\max }$ & $\leq 13 G y$ \\
\hline
\end{tabular}

(a) Planning constraints

\begin{tabular}{lll} 
Parameter & Symbol & Value \\
\hline Magnetic field & $B_{0}$ & $1.5 T$ \\
\hline Photon energy & $E$ & $6 M V$ \\
\hline Angle increment & $\Delta \Phi$ & $24^{\circ}$ \\
\hline
\end{tabular}

(b) Physical parameters

Table 1: Planning constraints and parameterization for the created kidney case.

\subsection{Plan generation}

For this proof of concept, renal cell carcinoma (RCC) cases were generated by projecting fictional lesions into two volunteers' right kidneys. Additionally, both kidneys, myelum, liver, stomach, pancreas and bowels were identified as OAR and contoured as seen in figure 1 a.

Based on constraints previously published by Stam, van Vulpen, Barendrecht, Zonnenberg, Crijns, Lagendijk \& Raaymakers (2013a), a plan was generated using the parameters in table 1a. For this study, the planned isocenter was set to the center of the tumor. The planning goal was to deliver 25Gy to the target. A PTV-GTV margin of $2 \mathrm{~mm}$ was used to obtain the $80 \%$ isodose line at the border of the GTV $\left(D_{99, G T V}=20 G y\right)$. Fluence was optimized by in-house developed software using an iterative approach by Ziegenhein et al. (2013) using pre-calculated beamlets for 15 equally space beam angles (see table $1 \mathrm{~b}$ ). The MC-based beamlet generation (Hissoiny, Raaijmakers, Ozell, Després \& Raaymakers 2011) took the MR-linac magnetic field $B_{0}=1.5 T$ into account. Finally, the plans were sequenced into deliverable stepand-shoot intensity modulated radiotherapy (IMRT) segments using the adaptive 


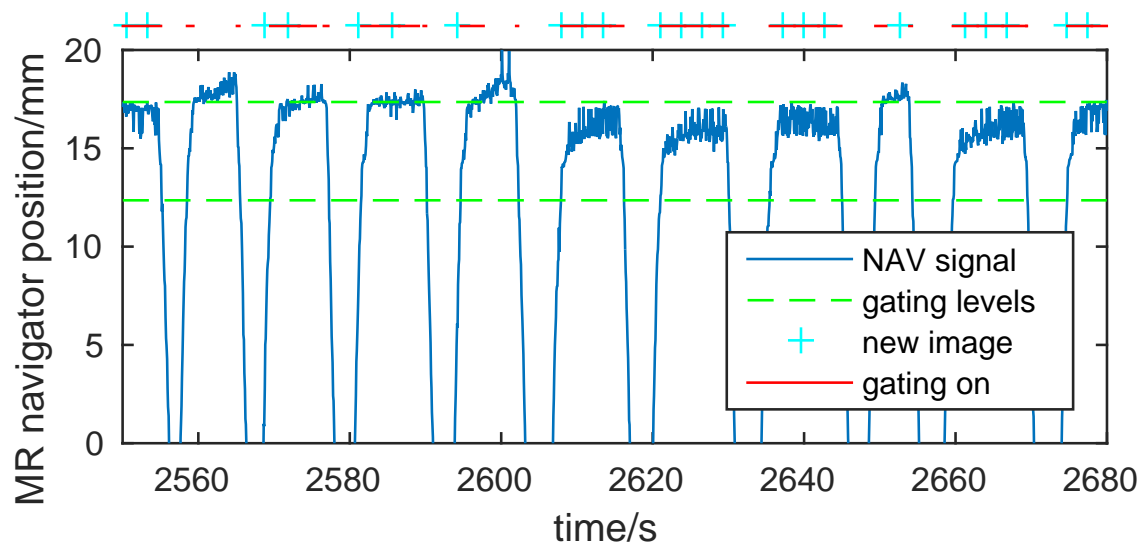

Figure 4: Navigator data with acceptance window: between the gating levels, imaging and beam generation becomes active. While the MR volumes are sampled, the plan segments are gradually delivered according to the treatment plan and the gating intervals. The high gating efficiency $(\approx 53 \%)$ is clearly recognizable.

sequencing (ASEQ) method (Kontaxis et al. 2015).

\subsection{Plan delivery}

Subsequently, the generated plan was delivered on a clinical linac emulator (Elekta AB, Stockholm, Sweden). The emulator featured an Agility 160 multileaf collimator (MLC) (Elekta AB, Stockholm, Sweden) and a realistic model of the gantry and beam generation. During the entire delivery the machine parameters were logged with a sampling interval of $40 \mathrm{~ms}$. This step was required to synchronise the exact machine status (leaf position, diaphragm position, gantry angle, dose rate) to the respectively valid anatomy.

\subsection{Pseudo-CT definition and generation}

Generally, for the planning stage, information about the electron density is needed for the fluence optimization. However, since CT data acquisition of the (healthy) volunteers was not feasible, pCTs were synthesized. Based on a two-component model (Kerkhof et al. 2010, Stam, van Vulpen, Barendrecht, Zonnenberg, Crijns, Lagendijk \& Raaymakers 2013a), the body outline was extracted from the reference MR image. Subsequently the body was filled with the Hounsfield units (HUs) of water $H U_{\text {water }}=0$, while the surrounding air was set to the HU of air $H U_{\text {air }}=-2900$. This yielded the two-component $\mathrm{pCT}$.

Subsequently, the push-forward DVF $V^{-1}$ extracted from the dynamic data (figure 2a ) was used to transform the planning-pCT to the new dynamic grid (figure 2b). This yielded a pCT for every dynamic, $p C T_{n}$.

\subsection{Dose reconstruction}

In this work, the navigator echo signal was used to trigger both imaging and the simulated treatment beam. For every $\mathrm{pCT}$ instance, an integration time $\Delta T_{n}$ is 
calculated by summing the valid gating intervals as depicted in Figure 4 . Together with the machine parameters extraction described in section section 2.5, a relation between momentaneous anatomy and machine state can be built up. Every acquired $3 D$ volume is thus valid for one or more individual segment(s).

Subsequently, using a MC based dose calculation (Hissoiny, Ozell, Bouchard \& Després 2011), dose is reconstructed on the respectively valid $p C T_{n}$ using the individual segment from the logged machine data (Luo et al. 2006). This produced dose maps $D_{n}$ for all acquired anatomies, which were eventually warped back to the original grid using $V_{n}$ (figure 2d).

For MC calculations, a computer with two 12-core (Intel Xeon E5-2695, Intel, Santa Clara, CA, USA) CPUs and a Tesla K20c (NVIDIA, Santa Clara, CA, USA) GPU was used. One segment took around $15 \mathrm{~s}$ to calculate with a $5 \%$ variance.

\subsection{Plan validation}

In order to validate the treatment plan, nominal (planned) doses were compared against the reconstructed doses. For error quantification, dose-volume histogram (DVH) points and integral parameters $\left(D_{\max }, D_{\text {mean }}, D_{\min }\right)$ were calculated for the target volumes and the surrounding organs at risk. Furthermore, planning constraints were compared between planned and actual delivery. To pinpoint local differences, dose difference maps were calculated and the respective maximal, mean and minimal deviation was extracted. 


\section{Results}

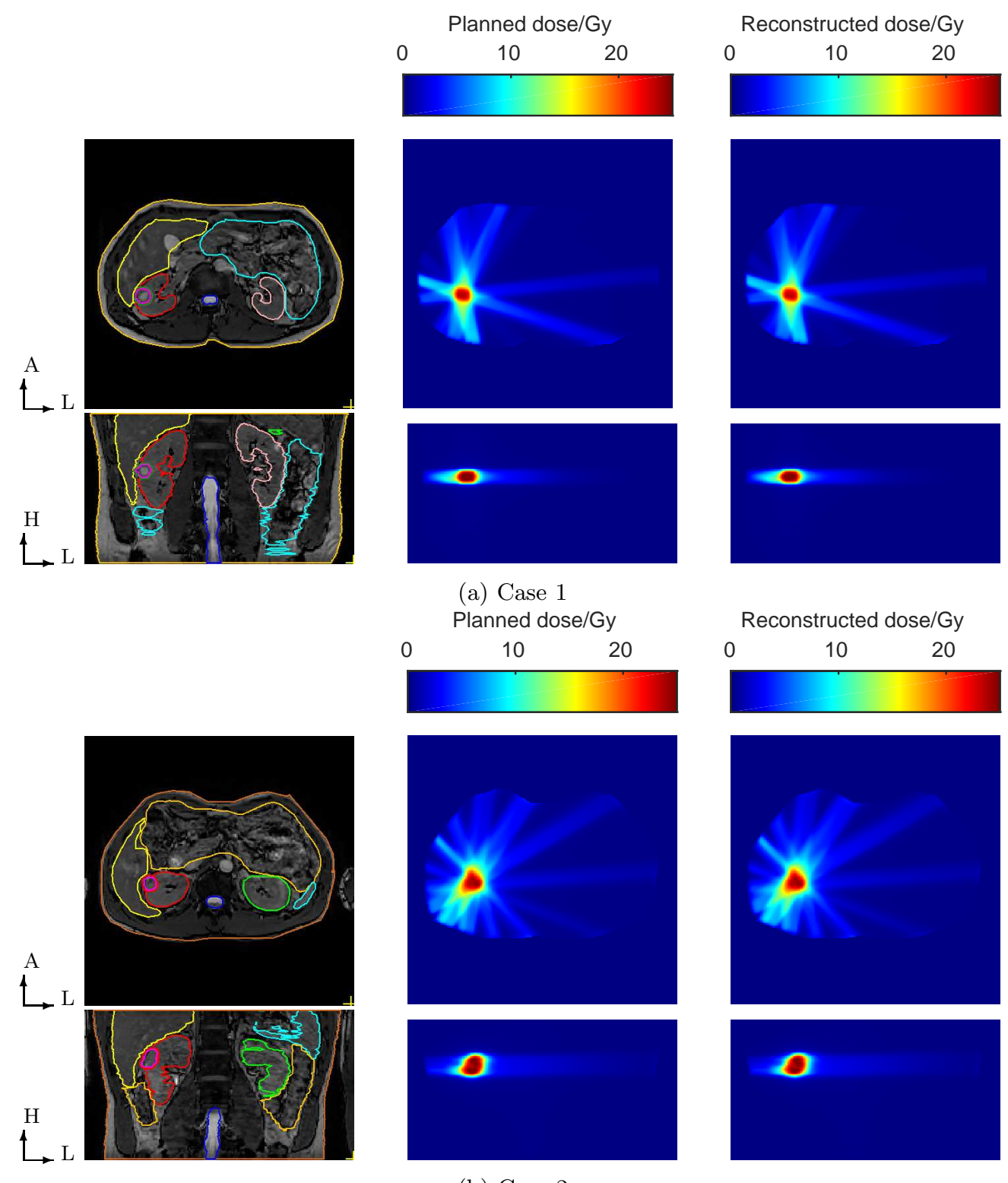

(b) Case 2

Figure 5: Cases 1 and 2 in figures (a) and (b), from left to right: selected slices (top row: transversal, bottom row: coronal) in delineated anatomy, the planned dose distribution and the accumulated dose incorporating the moving anatomies.

Anatomic directions: anterior $(\mathrm{A})$, head $(\mathrm{H})$ and left $(\mathrm{L})$.

Figure 5 depicts representative transverse slices of the anatomic reference images for both cases. The kidney lesions differ in location: case 1 is located in a more internal, inferior position as compared to case 2. The visual comparison between 
the planned dose on the rigid anatomy and the reconstructed dose based on variable anatomy shows no obvious deviation in the selected slice.

\begin{tabular}{|c|c|c|c|c|}
\hline Volume & Dose & static & dyn & $\operatorname{diff}(\%)$ \\
\hline GTV & $\mathrm{D}_{99} *$ & 19.670 & 19.490 & -0.9 \\
\hline \multirow{6}{*}{ PTV } & $\mathrm{D}_{\max } *$ & 26.230 & 26.460 & 0.9 \\
\hline & $\mathrm{D}_{\min }$ & 11.890 & 12.110 & 1.9 \\
\hline & $D_{\text {mean }}$ & 21.580 & 21.580 & 0.0 \\
\hline & $\Delta \mathrm{D}_{\max }$ & \multirow{2}{*}{\multicolumn{3}{|c|}{0.6}} \\
\hline & $\Delta D_{\min }$ & \multirow{2}{*}{\multicolumn{3}{|c|}{$\begin{array}{c}-0.7 \\
0.0 \\
\end{array}$}} \\
\hline & $\Delta D_{\text {mean }}$ & & & \\
\hline \multirow{7}{*}{ Kidney } & $\mathrm{D}_{\max }$ & 25.000 & 25.110 & 0.4 \\
\hline & $\mathrm{D}_{\min }$ & 0.080 & 0.070 & -12.5 \\
\hline & $D_{\text {mean }} *$ & 2.010 & 2.030 & 1.0 \\
\hline & $\mathrm{D}_{25} *$ & 1.216 & 1.268 & 4.3 \\
\hline & $\Delta \mathrm{D}_{\max }$ & \multirow{3}{*}{\multicolumn{3}{|c|}{$\begin{array}{c}0.6 \\
-0.6 \\
0.0\end{array}$}} \\
\hline & $\Delta \mathrm{D}_{\min }$ & & & \\
\hline & $\Delta D_{\text {mean }}$ & & & \\
\hline \multirow{7}{*}{ Liver } & $\mathrm{D}_{\max }$ & 20.400 & 20.470 & 0.3 \\
\hline & $\mathrm{D}_{\min }$ & 0.000 & 0.010 & - \\
\hline & $D_{\text {mean }}$ & 0.570 & 0.570 & 0.0 \\
\hline & $\mathrm{D}_{30 *}$ & 0.191 & 0.192 & 0.5 \\
\hline & $\Delta \mathrm{D}_{\max }$ & \multirow{3}{*}{\multicolumn{3}{|c|}{$\begin{array}{c}0.4 \\
-0.7 \\
0.0\end{array}$}} \\
\hline & $\Delta \mathrm{D}_{\min }$ & & & \\
\hline & $\Delta D_{\text {mean }}$ & & & \\
\hline \multirow{2}{*}{ Bowels } & $\mathrm{D}_{\max }$ & 1.800 & 1.760 & -2.2 \\
\hline & $\mathrm{D}_{5} *$ & 0.177 & 0.179 & 1.1 \\
\hline Stomach & $\mathrm{D}_{4} *$ & 0.033 & 0.033 & 0.0 \\
\hline Myelum & $\mathrm{D}_{\max } *$ & 1.390 & 1.430 & 2.9 \\
\hline
\end{tabular}

(a) Case 1

\begin{tabular}{|c|c|c|c|c|}
\hline Volume & Dose & static & dyn & $\operatorname{diff}(\%)$ \\
\hline GTV & $\mathrm{D}_{99 *}$ & 20.370 & 19.990 & -1.9 \\
\hline \multirow{6}{*}{ PTV } & $\mathrm{D}_{\max } *$ & 26.760 & 26.490 & -1.0 \\
\hline & $\mathrm{D}_{\min }$ & 13.810 & 13.890 & 0.6 \\
\hline & $D_{\text {mean }}$ & 21.990 & 21.750 & -1.1 \\
\hline & $\Delta D_{\max }$ & \multicolumn{3}{|c|}{1.5} \\
\hline & $\Delta D_{\min }$ & \multirow{2}{*}{\multicolumn{3}{|c|}{$\begin{array}{l}-2.3 \\
-0.2\end{array}$}} \\
\hline & $\Delta D_{\text {mean }}$ & & & \\
\hline \multirow{7}{*}{ Kidney } & $\overline{D_{\max }}$ & 24.530 & 24.290 & -1.0 \\
\hline & $\mathrm{D}_{\min }$ & 0.080 & 0.080 & 0.0 \\
\hline & $\mathrm{D}_{\text {mean }} *$ & 3.650 & 3.570 & -2.2 \\
\hline & $\mathrm{D}_{25 *}$ & 5.080 & 4.991 & -1.8 \\
\hline & $\Delta D_{\max }$ & \multicolumn{3}{|c|}{1.2} \\
\hline & $\Delta D_{\min }$ & \multirow{2}{*}{\multicolumn{3}{|c|}{$\begin{array}{l}-2.5 \\
-0.1\end{array}$}} \\
\hline & $\Delta D_{\text {mean }}$ & & & \\
\hline \multirow{7}{*}{ Liver } & $\mathrm{D}_{\max }$ & 19.990 & 20.330 & 1.7 \\
\hline & $\mathrm{D}_{\min }$ & 0.020 & 0.020 & 0.0 \\
\hline & $\mathrm{D}_{\text {mean }}$ & 0.890 & 0.910 & 2.2 \\
\hline & $\mathrm{D}_{30} *$ & 0.221 & 0.233 & 5.4 \\
\hline & $\Delta D_{\max }$ & \multirow{3}{*}{\multicolumn{3}{|c|}{$\begin{array}{c}1.9 \\
-1.3 \\
0.0\end{array}$}} \\
\hline & $\Delta \mathrm{D}_{\min }$ & & & \\
\hline & $\Delta \mathrm{D}_{\text {mean }}$ & & & \\
\hline \multirow{2}{*}{ Bowels } & $\mathrm{D}_{\max }$ & 17.470 & 16.870 & -3.4 \\
\hline & $\mathrm{D}_{5} *$ & 2.423 & 2.419 & -0.2 \\
\hline Stomach & $\mathrm{D}_{4} *$ & 0.087 & 0.087 & 0.0 \\
\hline Myelum & $D_{\text {max }} *$ & 1.670 & 1.650 & -1.2 \\
\hline
\end{tabular}

(b) Case 2

Table 2: DVH points and difference statistics for treatment validation of both cases. For each VOI, DVH-points $\left(D_{x x}\right)$, spatial integral parameters of absolute doses $\left(D_{\max }\right.$, $\left.D_{\text {mean }}, D_{\text {min }}\right)$ and voxel-wise differences $\left(\Delta D_{\max }, \Delta D_{\min }, \Delta D_{\text {mean }}\right)$ are shown. Planning constraints are marked with an asterisk $\left(^{*}\right)$.

The global DVH-points shown in table 2 show similar features: all dose constraints defined in table $1 \mathrm{a}$ were satisfied both with the static planning case and after validation using the variable anatomy. Only kidney- $D_{25}$ of case 2 is close to its maximum dose of $5 G y$. However, neither in the nominal nor in the reconstructed dose, the constraint is significantly violated and is therefore considered as plan compliant.

For case 1 local differences in table 2a shows little variation when incorporating moving anatomy. This is consistent with the global DVH point for this case, showing only little differences between static and moving anatomy.

Regarding the parameters for the second case (table 2b) reveals discrepancies between the DVH points and the local differences. While the differences in PTV are only around $1 \%$, local underdosage exceed $2 G y$ with overdosages up to $1.5 G y$ in the PTV.

As the most vicinal OAR, the statistics for the liver show a similar pattern. Although, DVH points do not suggest changes in the actually deposed anatomy, locally variations are significant, ranging from -1.3 to $1.9 \mathrm{~Gy}$.

These differences in the liver-kidney region are equally prominent in the difference images of figure $6 \mathrm{~b}$. As primary source for the dose alteration, the beam at $216^{\circ}$ (posterior-anterior) induces high difference gradients in the kidney-liver transition 


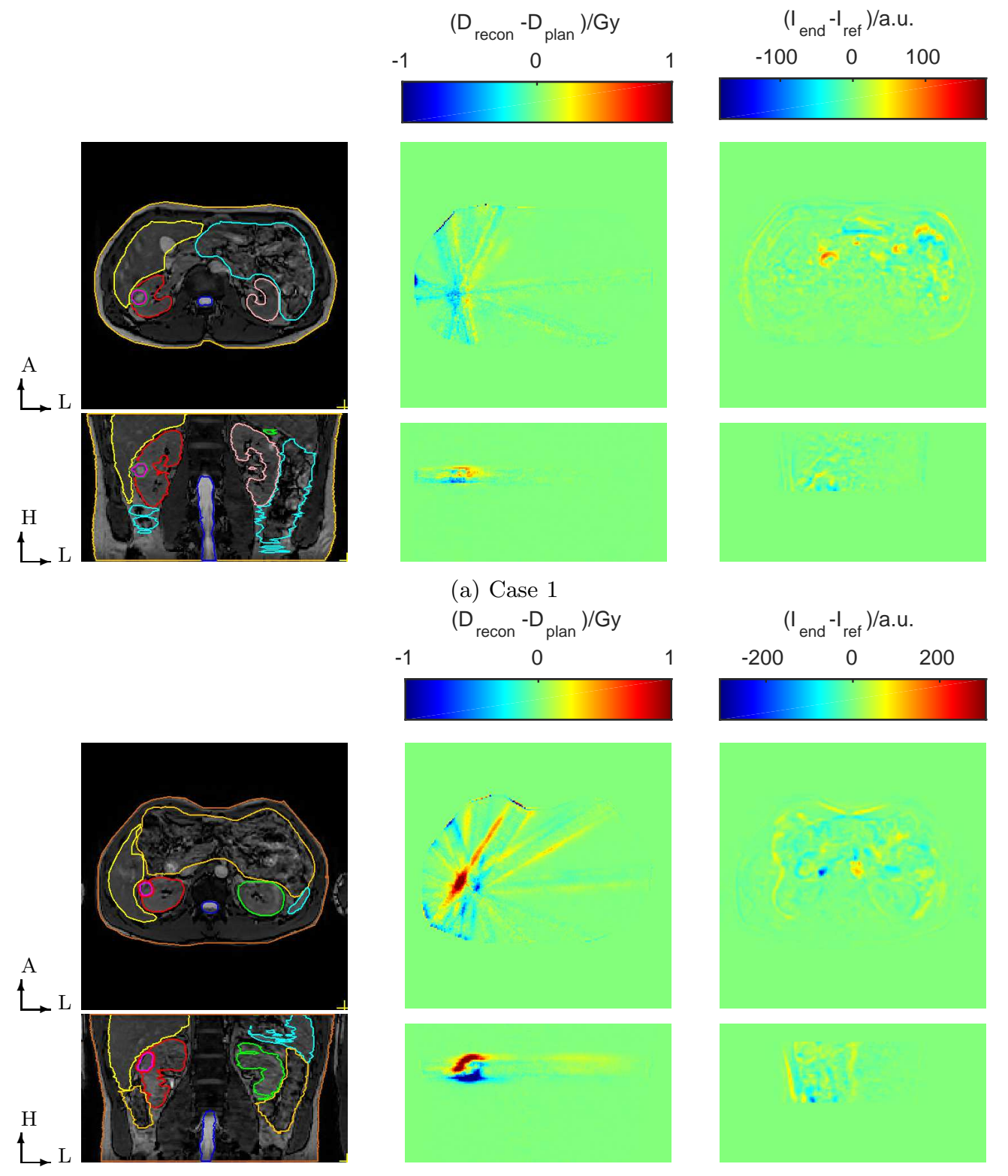

(b) Case 2

Figure 6: Visual dose and image comparison of both cases (a and b). Left: anatomic orientation, middle: dose differences between reconstructed delivery and planning $\left(D_{\text {recon }}-D_{\text {plan }}\right)$, right: difference between final and reference anatomy $\left(I_{\text {end }}-I_{\text {ref }}\right)$, indicating the anatomy displacements over the treatment time transversally (top row) and coronally (bottom row). 
region. Both underdosage and overdosage in this region is caused by anatomy variations in this beam angle. These variations are especially visible in the transverse and coronal dose profiles in figure $6 \mathrm{~b}$ and are correlated with the internal motion between kidney and liver. At the exiting portal of the same beam, the exit dose changed due to a variation of the diaphragm. Figure $6 \mathrm{a}$ as a comparison shows the same exit dose effects for the posterior entering beams. However, effects in the vicinity of the GTV are minor.

\section{Discussion}

Radiotherapy of mobile sites requires treatment validation. This work pursues a technique for on-line dose tracking for the treatment of abdominal sites using the on-line image capabilities of an MR-linac. We successfully devised and implemented a treatment pipeline capable of reconstructing the dose deposition in kidney cases using on-line MR imaging during treatment. By use of fast MR-navigator echoes, the abdomen was decoupled from breathing excursions. Entering the gating window triggered both treatment beam and $3 D$ image acquisition, the image covering an entire transverse slab around the target volume.

The continuous $3 D$ acquisitions can be utilized manifold: Firstly, deposited dose can be calculated integrally for an entire fraction. This enables the option to adapt subsequent fractions according to the intermediate deposition. Also, particularly erroneous parts of delivery causing high dose differences can be traced back in retrospect. Delivery elements which turn out to systematically induce larger errors in the plan validations can be identified and avoided for the sensitive population.

Secondly, when performing real-time accumulation after each imaging volume, local dosimetric or geometric errors at OAR can be used to trigger exception handling, based on predefined levels.

The two cases processed in this work suggest that inter-patient variations are significant. While for case 1 no considerable dose differences were found locally, the second case revealed considerable local differences. Most remarkably, the integral DVH points did not significantly change at any time. This suggests that integral measures ( $\gamma$, DVH points) alone are insufficient to characterize the validity of the plan outcome in a dosimetric sense.

Evidently, using on-line $3 D$ MR imaging for the dose reconstruction resolves the necessity to model anatomic motion as a bulk translation according to a surrogate signal, as described by Poulsen et al. (2012).

Compared to electronic portal imaging device (EPID) dosimetry as described for example by Lin et al. (2012), the presented method resolves the entire beam path, rather than projecting back over an integral pathway. Additionally, projective dosimetry relies on previously (retrospectively) acquired patient anatomies. Using EPIDs in the static $B$-field of an MR-system, the dosimetry would heavily rely on MCgenerated models of the beam's trajectory, in order to account for electron return effects (EREs) (Raaijmakers et al. 2005) at tissue-air boundaries.

In contrast, with the proposed pipeline, anatomy information is available on-line. Using the gated approach, anatomical drifts and other cycle-to-cycle variations, e.g. shown by Seregni et al. (2015), do not corrupt the anatomical accuracy. Instead of inferred data from a retrospective $4 D$ model as discussed by Sawant et al. (2014), true $3 D$ data is sampled. This yields accurate data representing the momentary state of anatomy. 
For a potential clinical deployment, the influence of target motion within the gating window has to be taken into account as shown by Stam, van Vulpen, Barendrecht, Zonnenberg, Crijns, Lagendijk \& Raaymakers (2013b). This can be accomplished by trading off gating window width against a residual motion margin.

Since the EPI readout in the imaging sequence is prone to geometric distortions, investigations of distortions correction methods will be necessary. The latter were already addressed by, e.g., Dragonu et al. (2009) for real-time high intensity focused ultrasound (HIFU) interventions. Moreover, to overcome image distortions, highbandwidth fast field echo (FFE) sequences will be considered, despite their contrast and artifact behavior, which have an adverse effect on the image registration.

The gating efficiency was estimated to around $50 \%$ for both cases, which would translate into an approximately two-fold treatment time. To address the problem of gating window drifts, we are currently evaluating gating level adaptation schemes. Additionally, ways to validate the dose reconstruction workflow itself are currently under investigation at our institution. Furthermore, ongoing efforts are translating the current off-line implementation into an on-line pipeline, deployable on the MRlinac.

\section{Conclusion}

We successfully implemented and demonstrated a workflow to perform a respiratory motion compensated, fully reconstructed treatment for abdominal site using the online imaging capabilities of an MR-linac. This proof of concept is a step towards the clinical implementation of dose validation using such a device.

\section{Acknowledgements}

The authors thank the ITEA (project 12026, SoRTS), the European Research Council (project ERC-2010-AdG-20100317, Sound Pharma) and Elekta AB (Stockholm, Sweden) for funding. 
On-line MR Imaging for Dose Validation of Abdominal Radiotherapy

\section{References}

Borst G R, Sonke J J, Betgen A, Remeijer P, van Herk M \& Lebesque J V 2007 Kilo-voltage conebeam computed tomography setup measurements for lung cancer patients; first clinical results and comparison with electronic portal-imaging device. Int. J. Radiat. Oncol. Biol. Phys. 68(2), 555-561.

Boye D, Lomax T \& Knopf A 2013 Mapping motion from 4D-MRI to 3D-CT for use in 4D dose calculations: a technical feasibility study. Med. Phys. 40(6), 061702.

Bussels B, Goethals L, Feron M, Bielen D, Dymarkowski S, Suetens P \& Haustermans K 2003 Respiration-induced movement of the upper abdominal organs: A pitfall for the threedimensional conformal radiation treatment of pancreatic cancer Radiother. Oncol. 68(1), 6974.

Crijns S P M, Raaymakers B W \& Lagendijk J J W 2012 Proof of concept of MRI-guided tracked radiation delivery: tracking one-dimensional motion. Phys. Med. Biol. 57(23), 7863-72.

De Meerleer G, Khoo V, Escudier B, Joniau S, Bossi A, Ost P, Briganti A, Fonteyne V, Van Vulpen M, Lumen N, Spahn M \& Mareel M 2014 Radiotherapy for renal-cell carcinoma Lancet Oncol. 15(4).

Denis De Senneville B, Mougenot C \& Moonen C T W 2007 Real-time adaptive methods for treatment of mobile organs by MRI-controlled high-intensity focused ultrasound Magn. Reson. Med. $\mathbf{5 7}(2), 319-330$.

Dragonu I, De Senneville B D, Quesson B, Moonen C \& Ries M 2009 Real-time geometric distortion correction for interventional imaging with Echo-planar imaging (EPI) Magn. Reson. Med. 61(4), 994-1000.

Fallone B G 2014 The Rotating Biplanar Linac-Magnetic Resonance Imaging System Semin. Radiat. Oncol. 24(3), 200-202.

Glitzner M, Crijns S P M, de Senneville B D, Lagendijk J J W \& Raaymakers B W 2015 On the suitability of Elektas Agility 160 MLC for tracked radiation delivery: closed-loop machine performance Phys. Med. Biol. 60(5), 2005-2017.

Haacke E M, Brown R W, Thompson M R \& Venkatesan R 1999 Magnetic Resonance Imaging: Physical Principles and Sequence Design Wiley.

Heinrich M P, Jenkinson M, Bhushan M, Matin T, Gleeson F V, Brady S M \& Schnabel J a 2012 MIND: Modality independent neighbourhood descriptor for multi-modal deformable registration Med. Image Anal. 16(7), 1423-1435.

Hissoiny S, Ozell B, Bouchard H \& Després P 2011 GPUMCD: A new GPU-oriented Monte Carlo dose calculation platform. Med. Phys. 38(2), 754-764.

Hissoiny S, Raaijmakers a J E, Ozell B, Després P \& Raaymakers B W 2011 Fast dose calculation in magnetic fields with GPUMCD. Phys. Med. Biol. 56(16), 5119-5129.

Horn B K \& Schunck B G 1981 Determining optical flow Artif. Intell. 17(1-3), 185-203.

ICRU 2010 ICRU 83: 4. Definition of volumes J. ICRU 10(1), 41-53.

Keall P J, Barton M \& Crozier S 2014 The Australian Magnetic Resonance Imaging-Linac Program Semin. Radiat. Oncol. 24(3), 203-206.

Keall P J, Cattell H, Pokhrel D, Dieterich S, Wong K H, Murphy M J, Vedam S S, Wijesooriya K \& Mohan R 2006 Geometric accuracy of a real-time target tracking system with dynamic multileaf collimator tracking system Int. J. Radiat. Oncol. Biol. Phys. 65(5), 1579-1584.

Keall P J, Colvill E, O'Brien R, Ng J A, Poulsen P R, Eade T, Kneebone A \& Booth J T 2014 The first clinical implementation of electromagnetic transponder-guided MLC tracking. Med. Phys. 41(2), 020702.

Keall P J, Kini V R, Vedam S S \& Mohan R 2001 Motion adaptive x-ray therapy: a feasibility study. Phys. Med. Biol. 46(1), 1-10.

Keall P, Vedam S, George R, Bartee C, Siebers J, Lerma F, Weiss E \& Chung T 2006 The clinical implementation of respiratory-gated intensity-modulated radiotherapy Med. Dosim. 31(2), 152-162.

Kerkhof E M, Balter J M, Vineberg K \& Raaymakers B W 2010 Treatment plan adaptation for MRI-guided radiotherapy using solely MRI data: a CT-based simulation study. Phys. Med. Biol. 55(16), N433-N440.

Kontaxis C, Bol G H, Lagendijk J J W \& Raaymakers B W 2015 Towards adaptive IMRT sequencing for the Phys. Med. Biol. 60, 2493-2509.

Kupelian P \& Sonke J J 2014 Magnetic Resonance-Guided Adaptive Radiotherapy: A Solution to the Future Semin. Radiat. Oncol. 24(3), 227-232.

Lagendijk J J W, Raaymakers B W \& van Vulpen M 2014 The Magnetic Resonance Imaging-Linac System Semin. Radiat. Oncol. 24(3), 207-209. 
Langen K M \& Jones D T L 2001 Organ motion and its management Int. J. Radiat. Oncol. Biol. Phys. 50(1), 265-278.

Lin M H, Li J, Wang L, Koren S, Fan J, Forkal E \& Ma C M 2012 4D patient dose reconstruction using online measured EPID cine images for lung SBRT treatment validation Med. Phys. 39(10), 5949.

Luo W, Li J, Price R a, Chen L, Yang J, Fan J, Chen Z, McNeeley S, Xu X \& Ma C M 2006 Monte Carlo based IMRT dose verification using MLC log files and R/V outputs. Med. Phys. 33(7), 2557-2564.

Mutic S \& Dempsey J F 2014 The ViewRay System: Magnetic Resonance-Guided and Controlled Radiotherapy Semin. Radiat. Oncol. 24(3), 196-199.

Østergaard Noe K, De Senneville B D, Elstrø m U V, Tanderup K \& Sø rensen T S 2008 Acceleration and validation of optical flow based deformable registration for image-guided radiotherapy. Acta Oncol. 47(7), 1286-1293.

Poulsen P, Schmidt M \& Keall P 2012 A method of dose reconstruction for moving targets compatible with dynamic treatments Med. Phys. 39(October), 6237-6246.

Raaijmakers a J E, Raaymakers B W \& Lagendijk J J W 2005 Integrating a MRI scanner with a 6 MV radiotherapy accelerator: dose increase at tissue-air interfaces in a lateral magnetic field due to returning electrons. Phys. Med. Biol. 50(7), 1363-1376.

Ravkilde T, Keall P J, Grau C, Hø yer M \& Poulsen P R 2014 Fast motion-including dose error reconstruction for VMAT with and without MLC tracking Phys. Med. Biol. 59(23), 7279.

Roujol S, Ries M, Moonen C \& Denis De Senneville B 2011 Automatic nonrigid calibration of image registration for real time MR-guided HIFU ablations of mobile organs IEEE Trans. Med. Imaging 30(10), 1737-1745.

Roujol S, Ries M, Quesson B, Moonen C \& De Senneville B D 2010 Real-time MR-thermometry and dosimetry for interventional guidance on abdominal organs Magn. Reson. Med. 63(4), 10801087.

Ruan D, Kupelian P \& Low D a 2011 Image-guided positioning and tracking. Cancer J. 17(3), 155-8.

Sawant A, Keall P, Pauly K B, Alley M, Vasanawala S, Loo B W, Hinkle J \& Joshi S 2014 'Investigating the feasibility of rapid MRI for image-guided motion management in lung cancer radiotherapy.'.

Schwartz L H, Richaud J, Buffat L, Touboul E \& Schlienger M 1994 Kidney mobility during respiration. Radiother. Oncol. 32(1), 84-86.

Seregni M, Paganelli C, Fattori G, Summers P, Bellomi M, Baroni G \& Riboldi M 2015 in 'ISBI 2015 .

Shirato H, Shimizu S, Kunieda T, Kitamura K, Van Herk M, Kagei K, Nishioka T, Hashimoto S, Fujita K, Aoyama H, Tsuchiya K, Kudo K \& Miyasaka K 2000 Physical aspects of a real-time tumor-tracking system for gated radiotherapy Int. J. Radiat. Oncol. Biol. Phys. 48(4), 11871195.

Stam M K, van Vulpen M, Barendrecht M M, Zonnenberg B a, Crijns S P M, Lagendijk J J W \& Raaymakers B W 2013a Dosimetric feasibility of MRI-guided external beam radiotherapy of the kidney. Phys. Med. Biol. 58(14), 4933-41.

Stam M K, van Vulpen M, Barendrecht M M, Zonnenberg B a, Crijns S P M, Lagendijk J J W \& Raaymakers B W $2013 b$ Dosimetric feasibility of MRI-guided external beam radiotherapy of the kidney. Phys. Med. Biol. 58(14), 4933-41.

Stam M K, van Vulpen M, Barendrecht M M, Zonnenberg B a, Intven M, Crijns S P M, Lagendijk J J W \& Raaymakers B W 2013 Kidney motion during free breathing and breath hold for MR-guided radiotherapy. Phys. Med. Biol. 58(7), 2235-45.

Wang Y, Rossman P J, Grimm R C, Riederer S J \& Ehman R L 1996 Navigator-echo-based realtime respiratory gating and triggering for reduction of respiration effects in three-dimensional coronary MR angiography. Radiology 198(1), 55-60.

Wolthaus J W H, Schneider C, Sonke J J, van Herk M, Belderbos J S a, Rossi M M G, Lebesque J V \& Damen E M F 2006 Mid-ventilation CT scan construction from four-dimensional respirationcorrelated CT scans for radiotherapy planning of lung cancer patients Int. J. Radiat. Oncol. Biol. Phys. 65(5), 1560-1571.

Yang D, Li H, Low D a, Deasy J O \& El Naqa I 2008 A fast inverse consistent deformable image registration method based on symmetric optical flow computation. Phys. Med. Biol. 53(21), 6143-6165.

Ziegenhein P, Kamerling C P, Bangert M, Kunkel J \& Oelfke U 2013 Performance-optimized clinical IMRT planning on modern CPUs. Phys. Med. Biol. 58(11), 3705-15. 